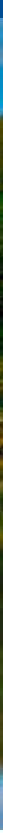

\title{
Longitudinal Effects in Space Charge Dominated Cooled Bunched Beams
}

Sergei Nagaitsev

Fermilab

Oct 5, 2017

This manuscript has been authored by Fermi Research Alliance, LLC under Contract No. DE-AC02-07CH11359 with the U.S. Department of Energy, Office of Science, Office of High Energy Physics. 


\section{Summary (in advance)}

- Longitudinal space-charge effects manifest themselves in several fascinating ways:

- Self-consistent stationary longitudinal distribution

- Synchrotron modes shifts with beam current

- These effects are easily accessible in low-energy electroncooled ion beams

- This talk is based on my research at the Indiana University Cyclotron Facility (IUCF) Cooler, but easily applicable to all other cooler rings 


\section{IUCF Cooler synchrotron (1988 - 2002)}
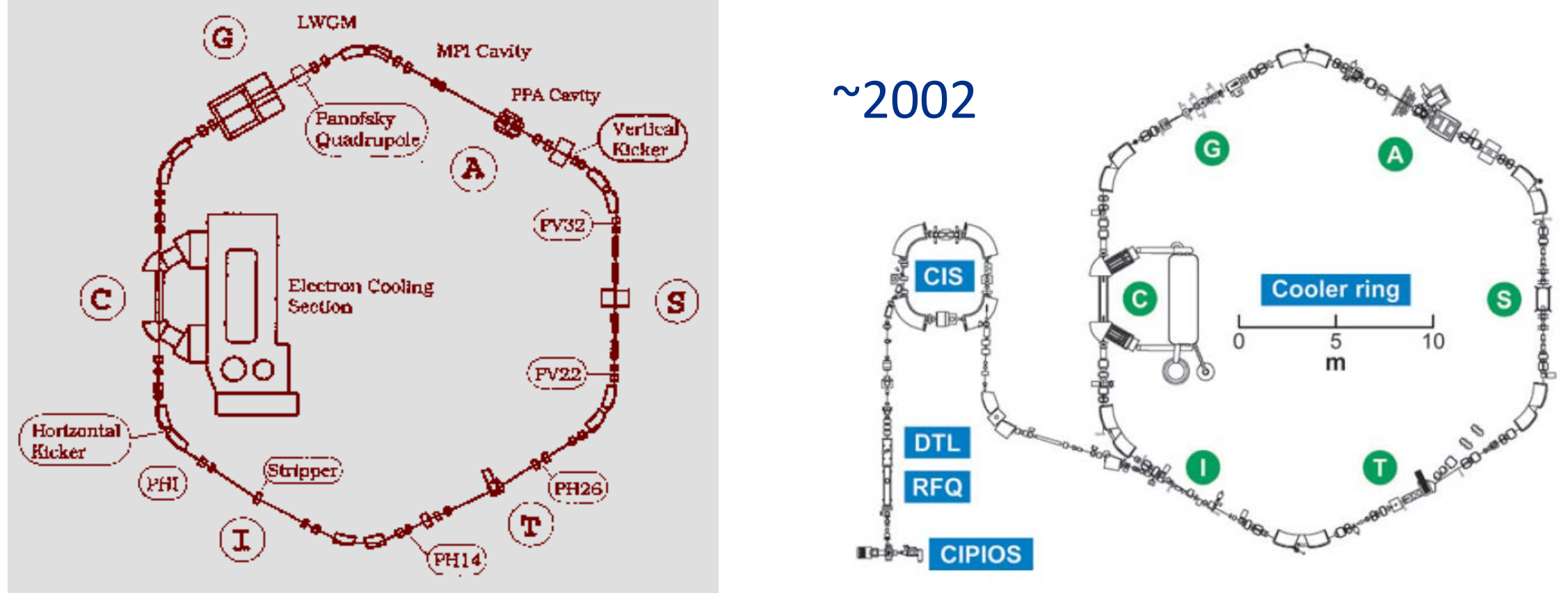

- These experiments were conducted with electron-cooled proton beams (45 - $150 \mathrm{MeV})$ below transition energy.

- Longitudinal and transverse profiles were measured for a range of beam currents. Also, the synchrotron dipole and quadrupole frequencies were measured. 


\section{Measurements}
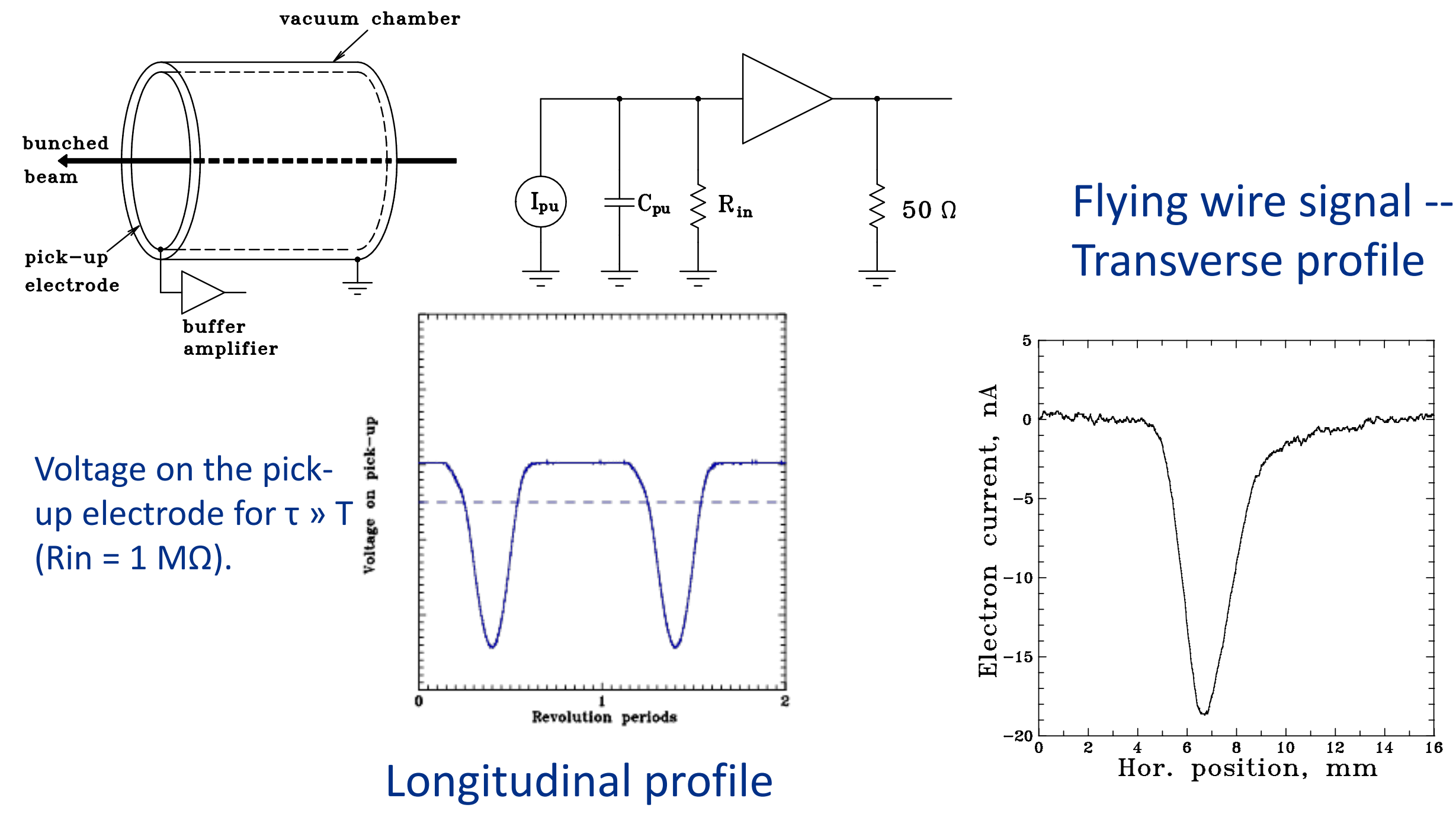
From measured long and transv beam profiles we can calculate the SC tune shifts:

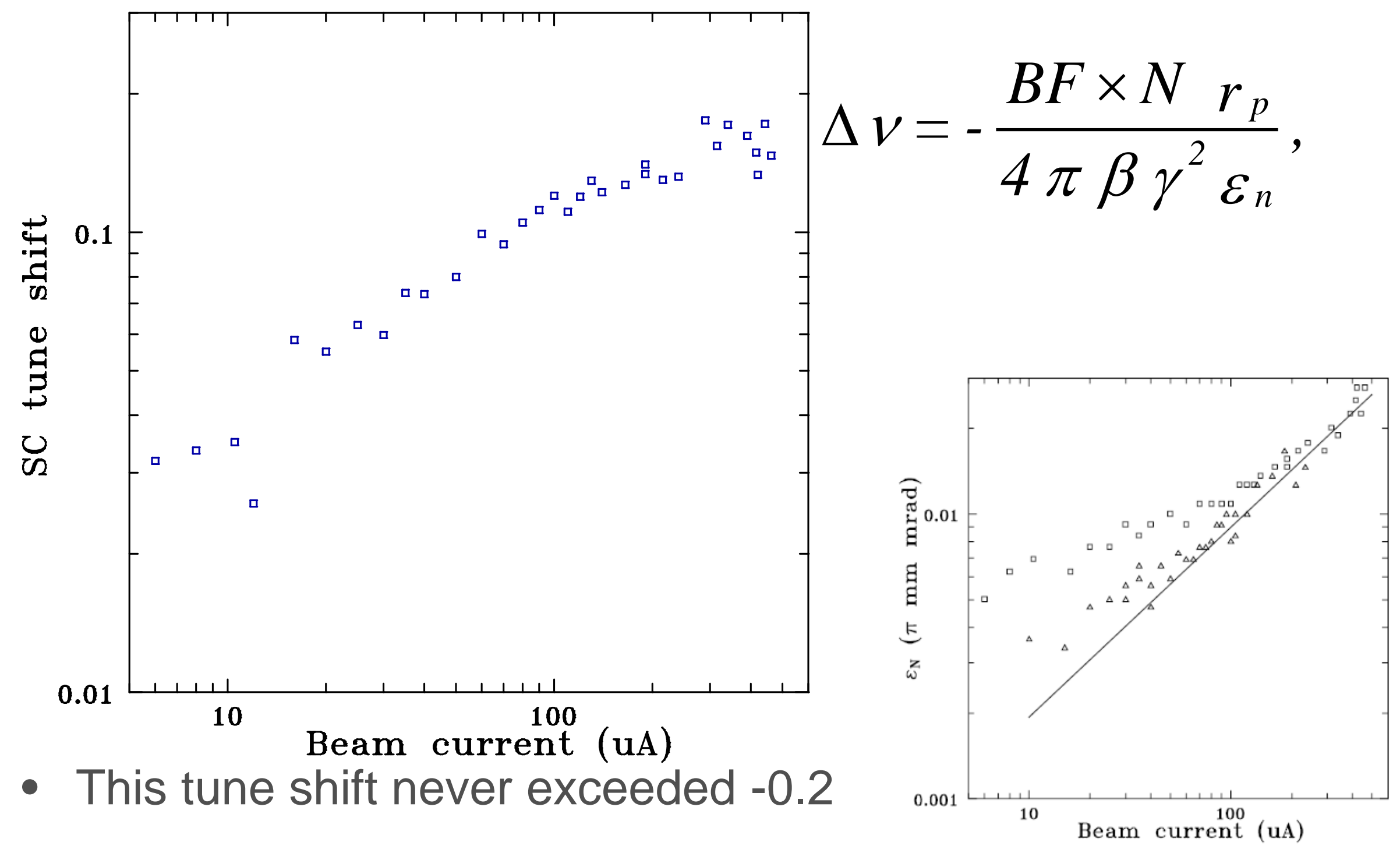

Figure 4. Normalized rms emittance as a function of the average bunched proton beam current before ( $\square$ ) and after $(\Delta)$ the alignment of electron and proton beams. Solid line is $I^{2 / 3}$. 


\section{...First, a quiz...}
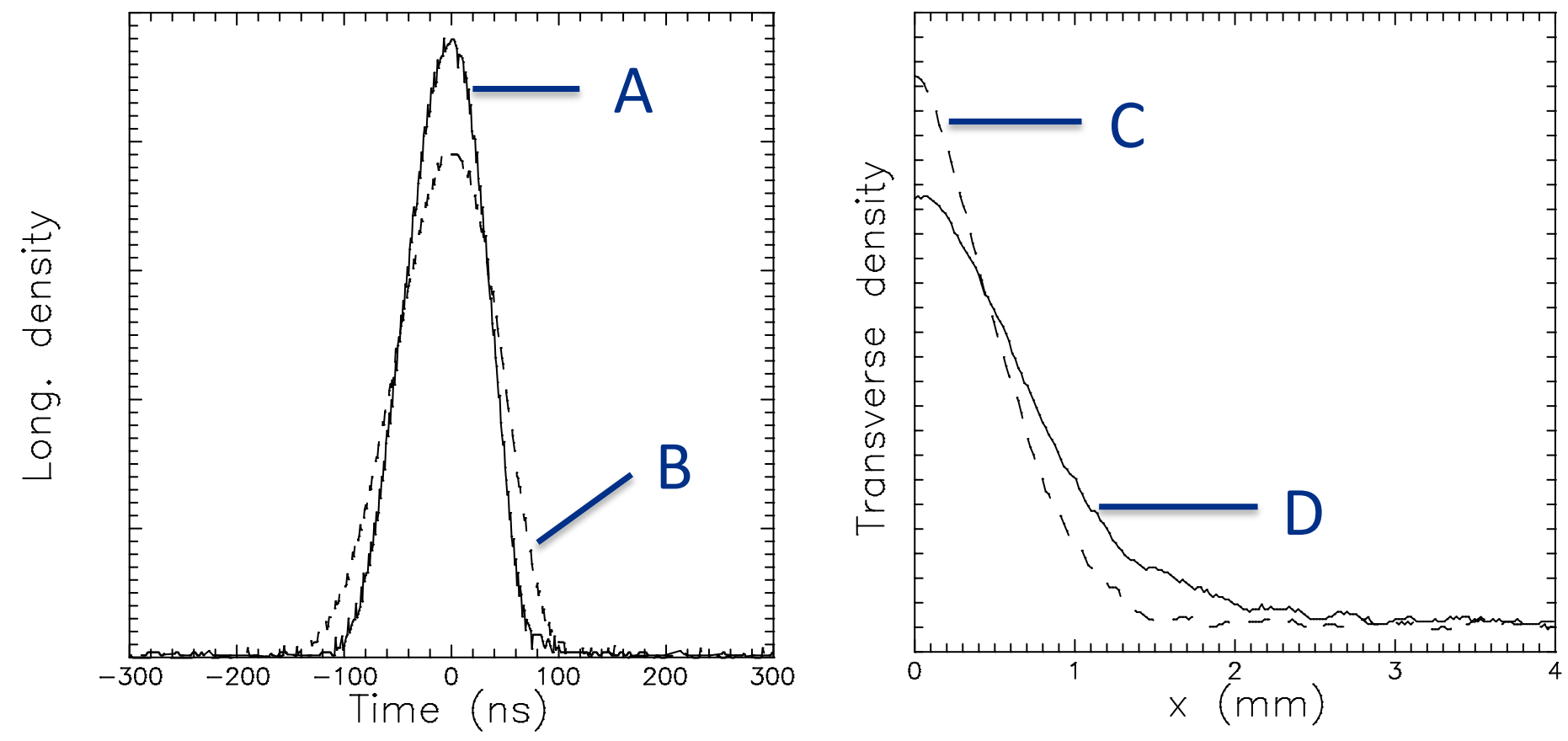

- These are measured stationary beam distribution profiles for a $150 \mathrm{MeV}$ proton beam with continuous electron cooling. One profile is taken with "good" vacuum, one with "bad" vacuum.

- Question: Which profiles correspond to "bad" vacuum?

- Bad vacuum: (1) A and C; (2) B and C; (3) A and D; (4) B and D 


\section{The correct answer is (3)}
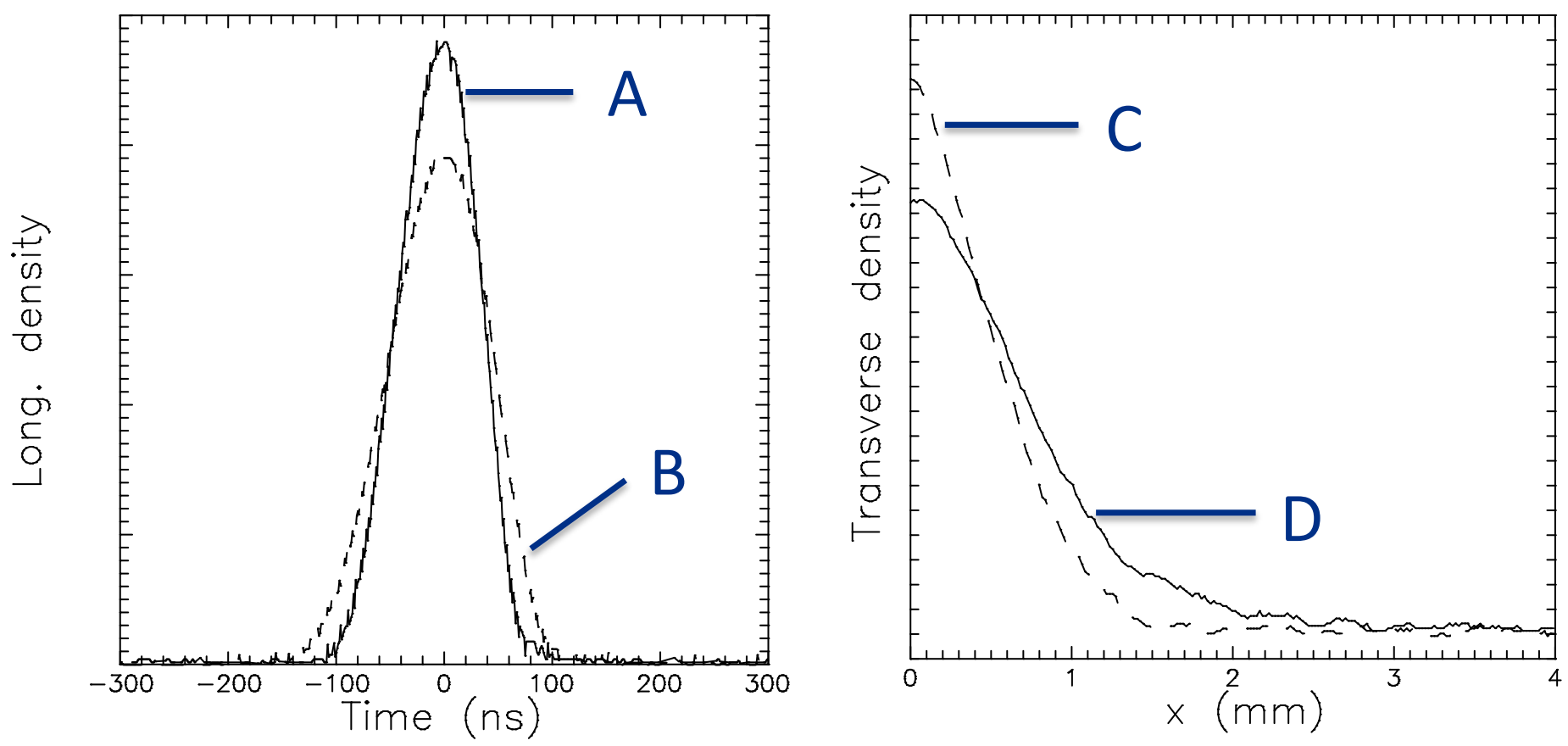

- (3) A and D;

- Transverse beam profile gets broader (D) from Coulomb scattering (because of "bad" vacuum) but the bunch length gets shorter $(A)$ because the longitudinal space-charge defocusing is reduced. 
LONGITUDINAL MOTION IN HIGH CURRENT ION BEAMS -

A SELF-CONSISTENT PHASE SPACE DISTRIBUTION

WITH AN ENYELOPE. EQUATION*

David Neuffer
Presented at the Particle Accelerator Conference, San Francisco, CA March 12-14, 1979

The ions in the bunch experience a space charge force given by

$$
F_{z}=\frac{-g}{\gamma^{2}} q^{2} e^{2} \frac{d \lambda}{d z}
$$

where $q$ is the ion charge state, $\lambda$ is the number of ions per unit length, and $g$ is a geometrical factor of order unity.

$$
\frac{\partial f}{\partial s}+z^{\prime} \frac{\partial f}{\partial z}+\left(-A \frac{\partial \lambda}{\partial z}-K z\right) \frac{\partial f}{\partial z}=0
$$

If we choose $f$ such that $f=f(H)$ then the Vlasov equation requires that $\frac{\partial f}{\partial S}=0$ and we have a stationary distribution. We must also choose an $f$ that is selfconsistent; that is

$$
\int f\left(z^{\prime}, z, s\right) d z^{\prime}=\lambda(z, s)
$$

As a simplest solution we desire $\lambda(z)$ to be parabolic:

$$
\begin{array}{ll}
\lambda=\frac{3}{4} \frac{N}{z_{m}}\left(1-\frac{z^{2}}{z_{m}^{2}}\right) \equiv \lambda_{0}\left(1-\frac{z^{2}}{z_{m}^{2}}\right) & |z|<z_{m} \\
\lambda=0 & |z|>z_{m}
\end{array}
$$




\section{With electron cooling, the Vlasov equation becomes the Fokker-Plank equation}

$$
\begin{gathered}
\frac{\partial \Psi}{\partial t}+\dot{\phi} \frac{\partial \Psi}{\partial \phi}+\dot{\delta} \frac{\partial \Psi}{\partial \delta}=\frac{\partial}{\partial \delta}\left(\lambda \delta \Psi+\frac{D}{2} \frac{\partial \Psi}{\partial \delta}\right), \\
\dot{\phi}=h \eta \omega_{o} \delta, \quad \dot{\delta}=f_{o} \frac{e V_{r f}}{\beta^{2} E} \sin (\phi)+\frac{Z_{o} g e^{2} N f_{o}}{\gamma^{2} \beta^{2} E} \frac{c h^{2}}{R} \frac{\partial \rho(\phi, t)}{\partial \phi}, \\
\rho(\phi, t)=\int_{-\infty}^{+\infty} \Psi(\phi, \delta, t) d \delta .
\end{gathered}
$$

For a uniform transverse distribution

$g=\log [$ chamber radius/beam radius] $+1 / 2$-- geometric factor

- This equation can be solved analytically for a stationary distribution function 


\section{The stationary distribution}

$$
\begin{gathered}
\Psi_{o}(\phi, \delta)=\frac{1}{(2 \pi)^{1 / 2} \sigma} e^{-\delta^{2} / 2 \sigma^{2}} \rho_{o}(\phi), \\
\rho_{o}(\phi) e^{-\alpha \rho_{o}(\phi)}=\rho_{o}(0) e^{-\alpha \rho_{o}(0)} \exp [\kappa(1-\cos (\phi))] .
\end{gathered}
$$

$\rho_{o}(0)$ must be chosen such that $\rho_{o}(\phi)$ is normalized to unity

$$
\alpha=\frac{Z_{o} g e^{2} N}{2 \pi \gamma^{2} \beta^{2} \sigma^{2} \eta E} \frac{c h}{R} ; \kappa=\frac{1}{2 \pi \sigma^{2} \beta^{2} \eta h} \frac{e V_{r f}}{E} .
$$

- There are two unknowns: $\sigma$ and $g$, the rms momentum spread and the geometric factor.

- For vanishing space-charge $(\alpha=0)$ the linear density $\rho_{o}(\phi)$ becomes Gaussian.

- For vanishing momentum spread $(\sigma \rightarrow 0)$ the linear density becomes

$$
\rho_{o}(\phi)=\frac{\gamma^{2}}{g h^{2}} \frac{V_{r f} R}{Z_{o} e N c}\left(\cos (\phi)-\cos \left(\phi_{o}\right)\right),
$$




\section{Stationary longitudinal bunch charge density}

distributions for a fixed momentum spread $(\sigma)$ and various bunch charges

\section{Charge density}

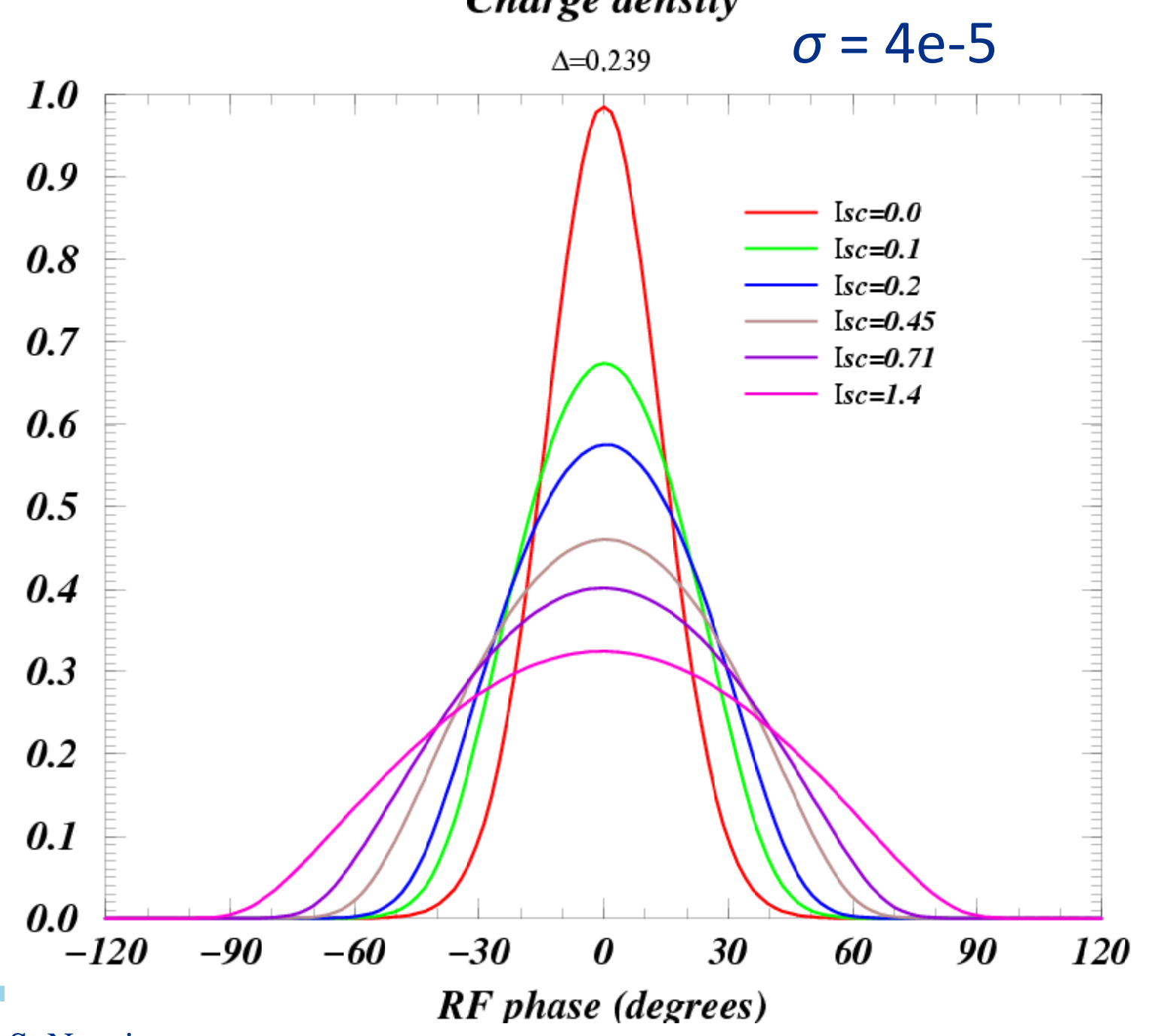




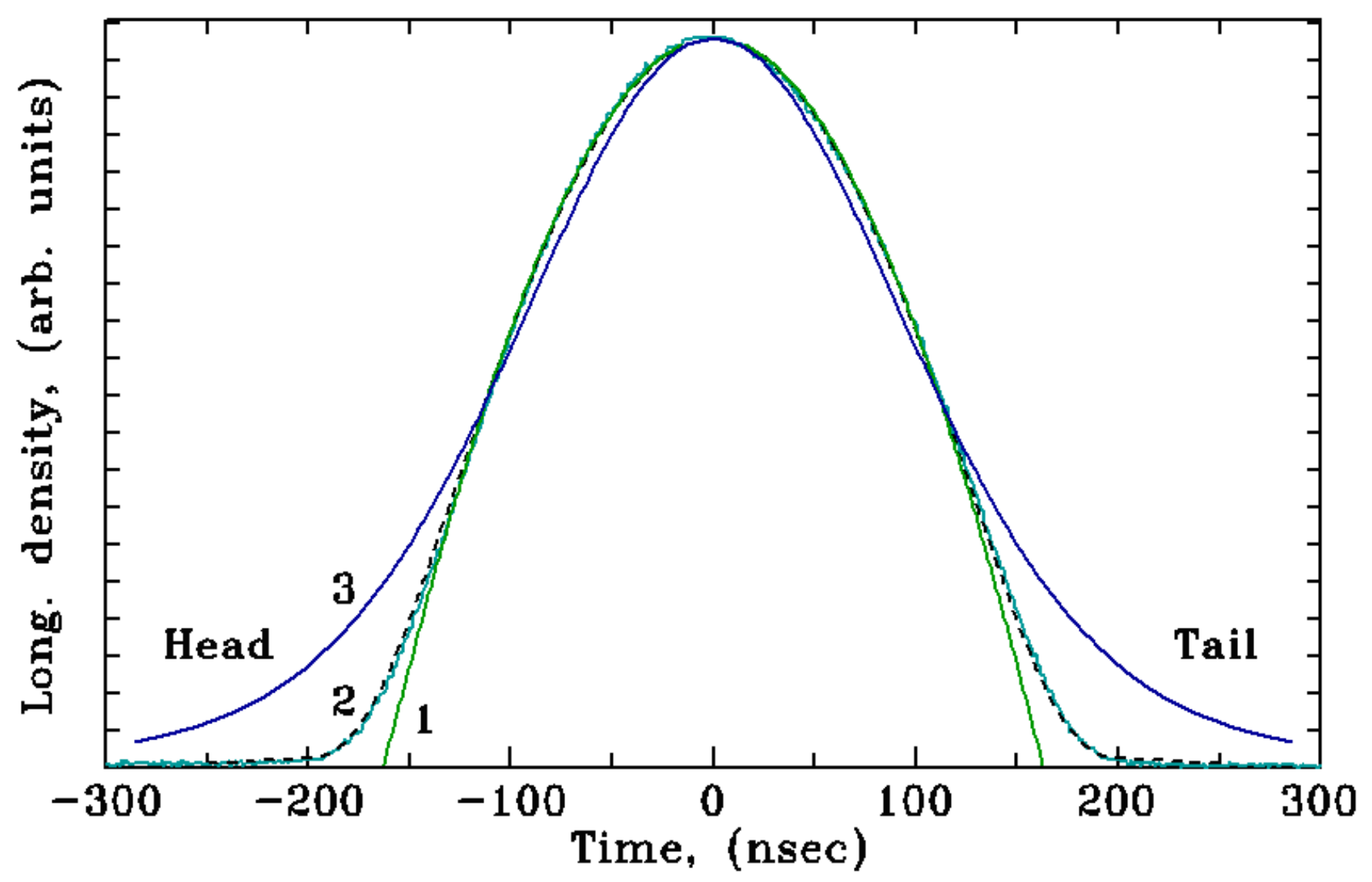

Measured (solid, 2) and theoretical (dashed, 2) linear density. Cosine (1) and Gaussian (3) fits are also presented. $I_{o} \approx 400 \mu \mathrm{A}$, $V_{\text {rf }} \approx 12$ V. IUCF Cooler, 1994 


\section{Cross-checking $g$ vs transverse beam radius}

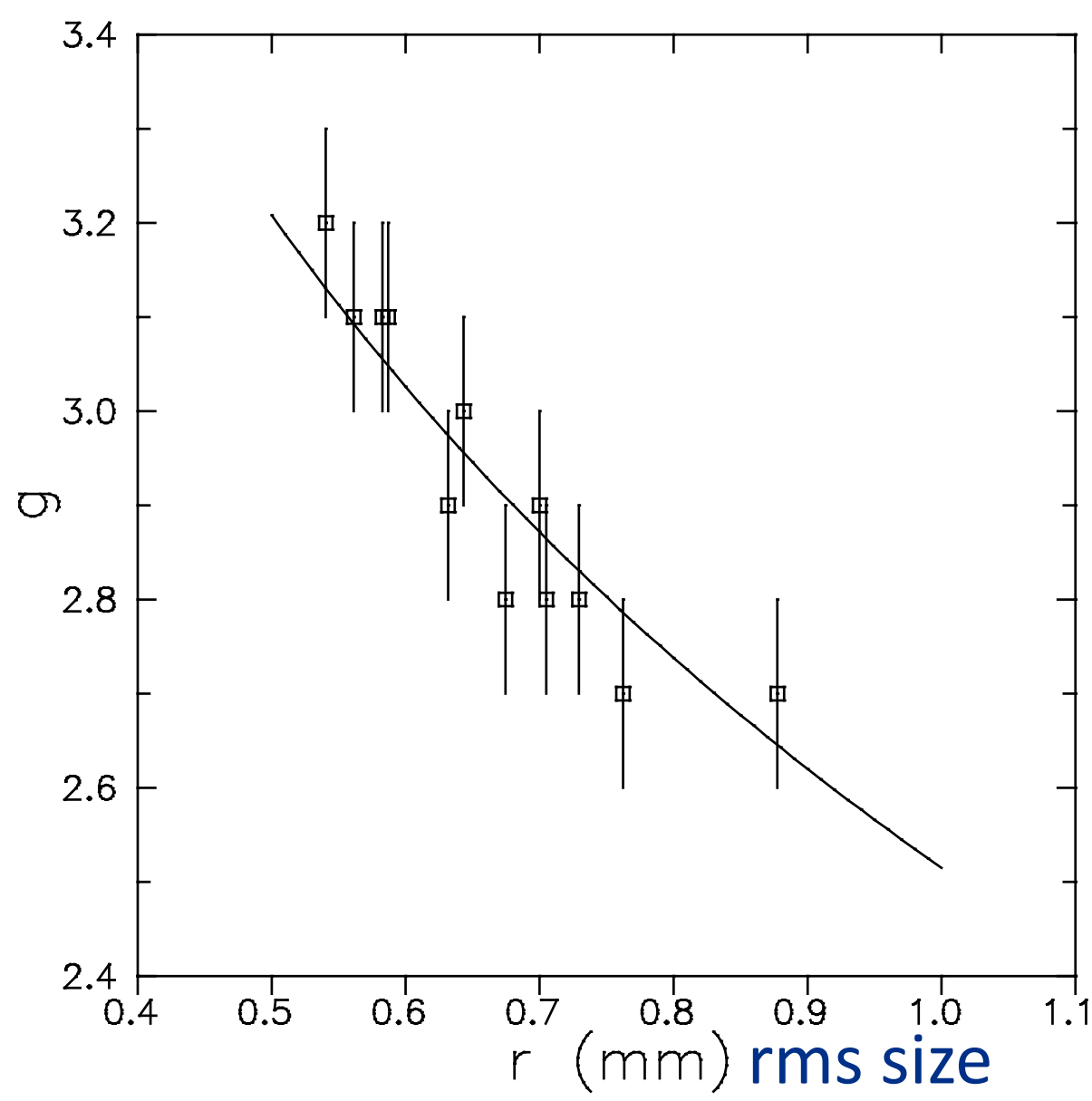

$$
g=\frac{1}{4}+\ln \left(\frac{b}{2 \times \text { rms size }}\right)
$$

- Best fit corresponds to $b \approx 20 \mathrm{~mm}$

- The IUCF cooler had the average beam pipe radius of $25 \mathrm{~mm}$ 


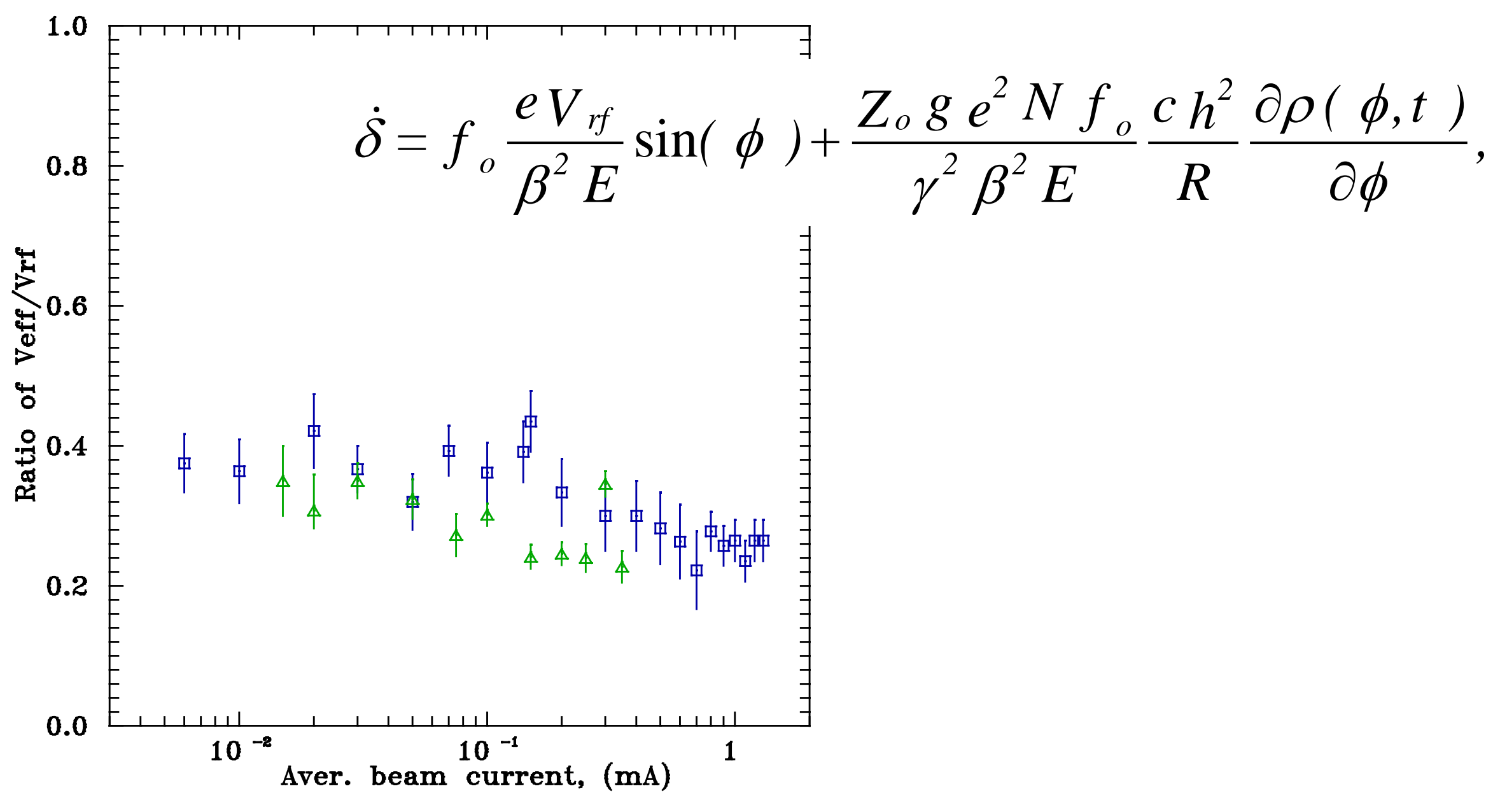

Ratio of the effective $\mathrm{rf}$ voltage to the applied rf voltage derived from the bunch shape fitting. $V_{I f}=12 \mathrm{~V}(\square), V_{r f}=126.4 \mathrm{~V}(\Delta)$. 


\section{Longitudinal dynamics of space-charge dominated} bunched beams

- Linear rf voltage model (from Neuffer's 1979 paper)

- Long envelope equation:

$$
\ddot{\phi}_{o}=\left(2 \pi h|\eta| f_{o}\right)^{2}\left(\frac{\varepsilon_{L}^{3}}{\phi_{o}^{3}}+\frac{N}{N_{o}} \frac{1}{\phi_{o}^{2}}-A \phi_{o}\right),
$$

- The dipole synchrotron frequency is not affected by SC

- After linearization, one obtains for the small-amplitude quadrupole (bunch length) oscillation frequency:

$$
\left(\frac{\omega_{q}}{\omega_{s o}}\right)^{2}=\frac{V_{\text {eff }}}{V_{r f}}+3 \text {. }
$$

- With no SC: $\omega_{q}=2 \omega_{\text {so }} \quad$-- it's a simple bunch rotation

- With zero momentum spread: $\omega_{q}=\sqrt{3} \omega_{\text {so }}$

- For the $\sin ()$ rf voltage the situation is more complicated 


\section{Long. bunch modes with sin() rf voltage}

- We found approx. analytic solutions for bunch modes with zero momentum spread. The modes are solutions to this equation

$$
\frac{d}{d \phi}\left(\frac{d \rho_{n}}{d \phi}\left(\cos (\phi)-\cos \left(\phi_{o}\right)\right)\right)+\left(\frac{\omega_{n}}{\omega_{s o}}\right)^{2} \rho_{n}=0 .
$$

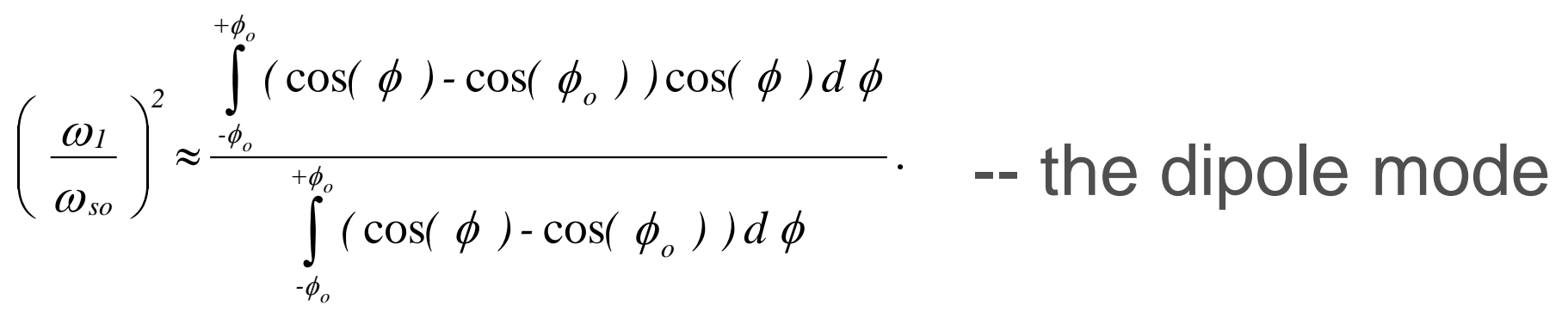

The quadrupole mode was found numerically

- For non-zero momentum spread we solved the timedependent F-P equation numerically

- Both modes were measured in the IUCF cooler 


\section{The dipole mode}
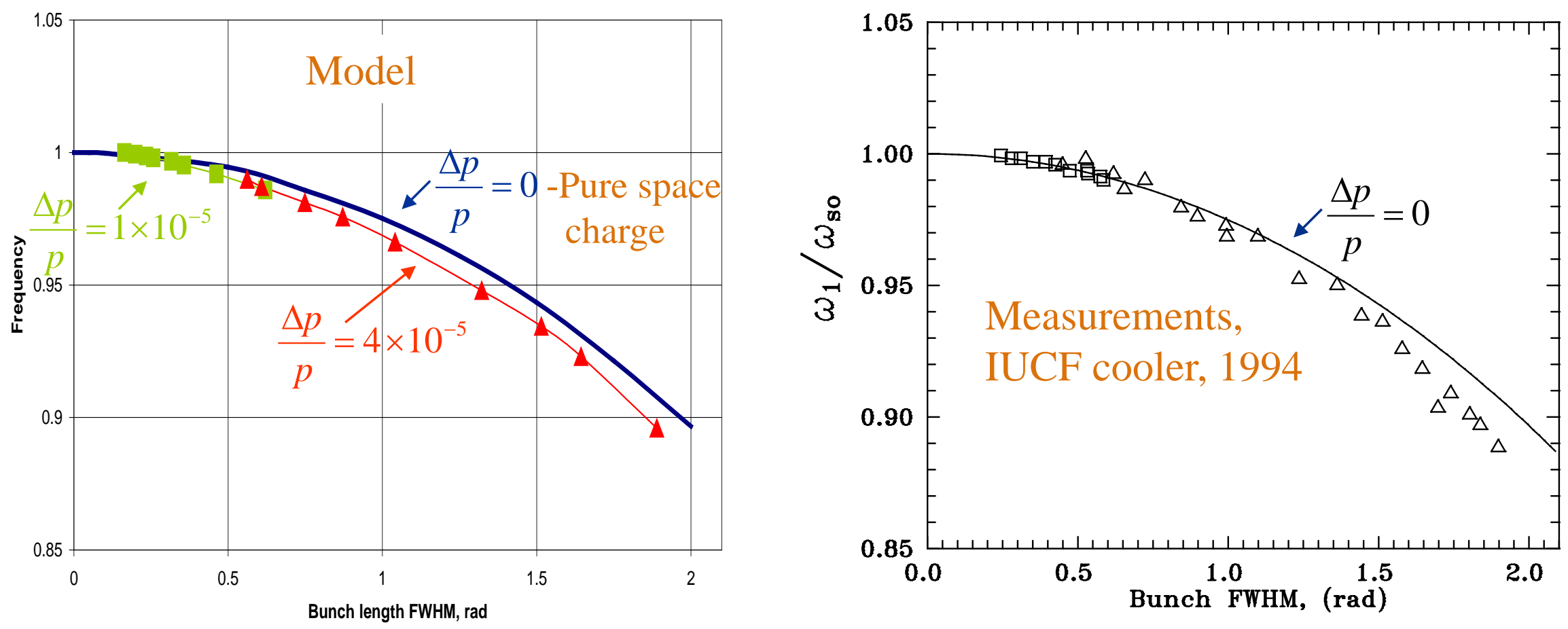

For a space-charge dominated regime the bunch length is primarily a function of a beam current, thus, for a sinusoidal rf voltage, the synchrotron frequency depends on the beam current! (solid lines are numerical models) 


\section{The quadrupole mode}

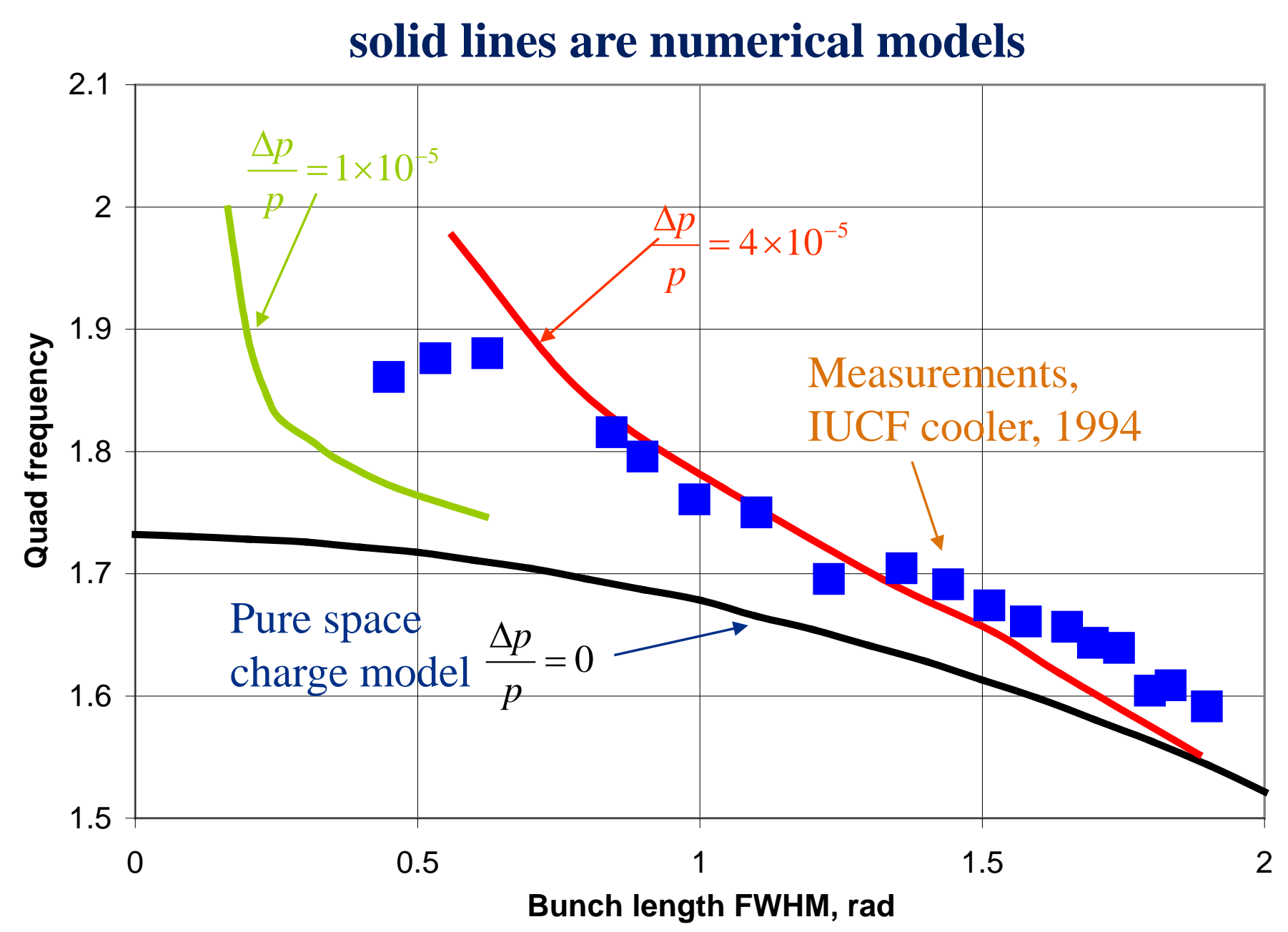

仵 Fermilab 


\section{Summary}

- The beam momentum spread can not be determined from the bunch length alone! Need to perform a fit with two parameters.

- Electron-cooled bunched beams are typically space-charge dominated longitudinally: the effective rf voltage amplitude in the bunch is small, $\sim 20 \%$ of external rf voltage.

- Need to take this into account in modeling of IBS, etc

- The coherent synchrotron (dipole) frequency depends on the beam current! Also, no decoherence in large amplitude synchrotron oscillations is observed. 